\title{
Neo-kaizen Applications on the Generic Operations Support and Renewal
}

\author{
K. Mori, N. Yoshikawa \\ Omron Corporation, Japan \\ Em:kenichiro_mori@omron.co.jp \\ K. Morita \\ Mitsui Engineering \& Shipbuilding Co., Ltd, Japan \\ T. Kimura, H. Goto \\ Japan Society for the Promotion of Machine Industry \\ M. Asamori, Y. Kamio \\ Toyo Engineering Corporation, Japan \\ Y. Fukuda \\ Hosei University, Japan
}

Keywords Model, Improvement, Operation support, Renewal, KAIZEN

\begin{abstract}
Conventional TQC (Total Quality Control) activities do not use information technologies adequately. The TQC activities adapted to a global manufacturing environment have been limited. This research deals with using information processing systems for the improvement of manufacturing systems based on a TQC method for a global manufacturing environment. We are developing the GOSR (General Operation Support and Renewal) in the IMS program. The G-OSR is a generic information architecture for operations support and renewal in the manufacturing. This paper describes to realize the KAIZEN demo scenarios based on the G-OSR and to show three prototype systems for applications. The application systems include KAIZEN support and remote monitoring support by information technologies.
\end{abstract}

\section{INTRODUCTION}


Continuous improvement activities for manufacturing systems are recognized more important than ever. Almost excellent companies carry out TQC (Total Quality Control) activities for the improvement of their processes to maintain the productivity for operating a manufacturing system. The activity is called KAIZEN activity in general.

However, conventional TQC activities have not been supported by information technologies compared with operation and maintenance [1][2][3]. The reasons are (1) lack of information models for the KAIZEN activity, (2) the cost of building an information system for the activity is higher than the benefit from the KAIZEN activity, and (3) operators or managers in the shop floor do not focus on their information systems as an improvement target.

In this paper, we propose the Neo-KAIZEN system to support continuous improvement based on an enhanced model and generic support system architecture. The system consists of data monitoring and logging from plants, statistical quality control assisting tools, simulation systems for evaluating the system performance, and operator support systems. The Internet and local area networks link all sub-systems.

Firstly, the KAIZEN activity and its extended model for a support system are proposed. Secondly, Generic Operations Support and Renewal (G-OSR) information architecture is described for a generic system design. Thirdly, Neo-KAIZEN systems based on the G-OSR are explained as application demonstrations. We discuss the performance and the improvement agility from the applications.

This research and development project has been supported by Global manufacturing toward 21century (Globeman-21) project and GLOBEMEN project in IMS International Research \& Development Program. The G-OSR is developed in the project based on the discussion with Australia, Europe and Japan partners.

\section{THE KAIZEN ACTIVITY MODEL AND ITS SUPPORT SYSTEM}

The basic method of continuous improvement (KAIZEN) system is TQC activity [4]. The purpose of TQC activity is to solve problems relating $\mathrm{Q}$ (Quality) C(Cost) $\mathrm{D}$ (date of delivery) S(Safety) M(Morale) on operating plants. All members in an enterprise from president to operators should join to TQC activity. The activities are carried out in all product life cycle such as market research, research and development, design, operation, and 
maintenance. The TQC activity consists of four processes, $\mathrm{P}(\mathrm{Plan}) \mathrm{D}(\mathrm{DO})$ $\mathrm{C}($ Check) A(Action).

To apply information technologies to the KAIZEN activity, PDCA process is extended to a new process model. As a result of analysis of basic models, we propose a spiral approach model for the KAIZEN activity support system (Neo-KAIZEN system) which has six phases instead of PDCA. Figure 1 shows the spiral approach model and the relation of PDCA process. Then the system is able to improve the QCDSM concerned with operation in the life cycle of manufacturing supported by information technologies. The six phases in Figure 1 are followings,

(1) Get Operation Status -The information concerned to operate the manufacturing systems is collected from the real shop floor.

(2) Analyse the Operation Status -The operation status of the manufacturing system is analysed based on the collected information.

(3) Find the Problems for the Operation -The major problems of the manufacturing system are found out from the result of analyses.

(4) Plan to Solve the Problem -The improvement proposal to solve the problems of the manufacturing system is planed.

(5) Evaluate the Plan -The proposal to solve the problems is evaluated in advance by simulation.

(6) Implement to the Process -The evaluated plan is implemented to the manufacturing system to improve the process.

Executing these six phases is repeated continuously on the Neo-KAIZEN system.

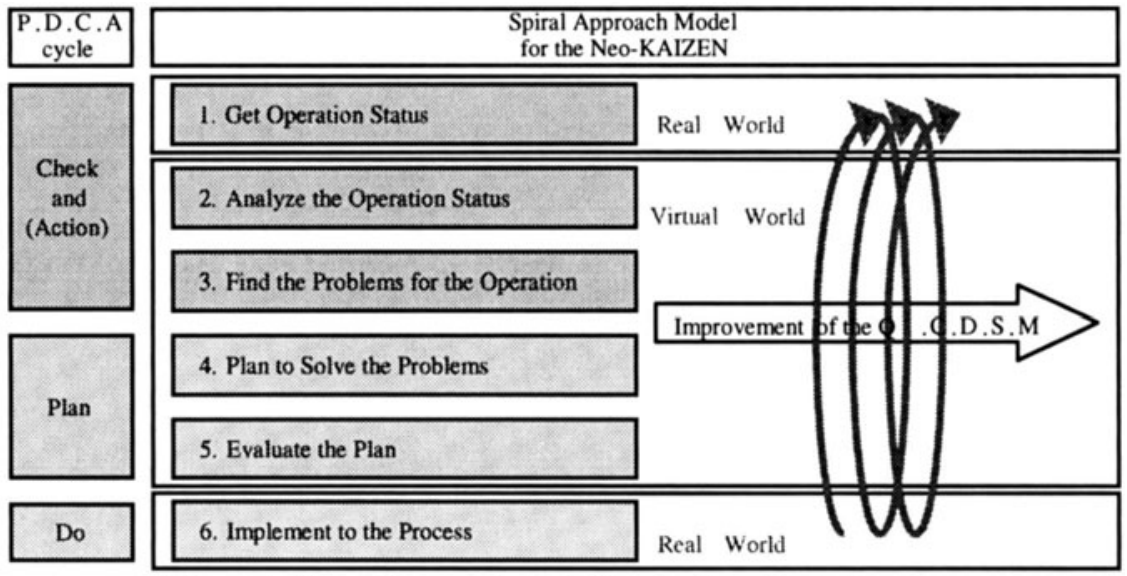

Figure 1 Spiral Approach Model for the Neo-KAIZEN system

\section{G-OSR INFORMATION ARCHITECTURE}


G-OSR (Generic Operations Support and Renewal) proposed here is a trial to realize a systematic approach to develop support systems of the processes of operations, maintenance, and renewal using the information technology. The objective of G-OSR is to develop a generic methodology for the common and cost effective design of the support systems [5].

The G-OSR architecture is defined based on the analysis of similar systems that supports remote operation and the KAIZEN activity. Additionally, the information infrastructure required to support systems is defined in the G-OSR architecture. The hierarchy of architecture and information technology corresponding to common applications and common tools are defined as the information infrastructure of G-OSR. G-OSR is visualized as a model that can make the use of the group of tools developed in this study, thus facilitating simplified integration with future systems. In addition, the groups of tools developed in the Neo-KAIZEN system are mapped to the G-OSR architecture.

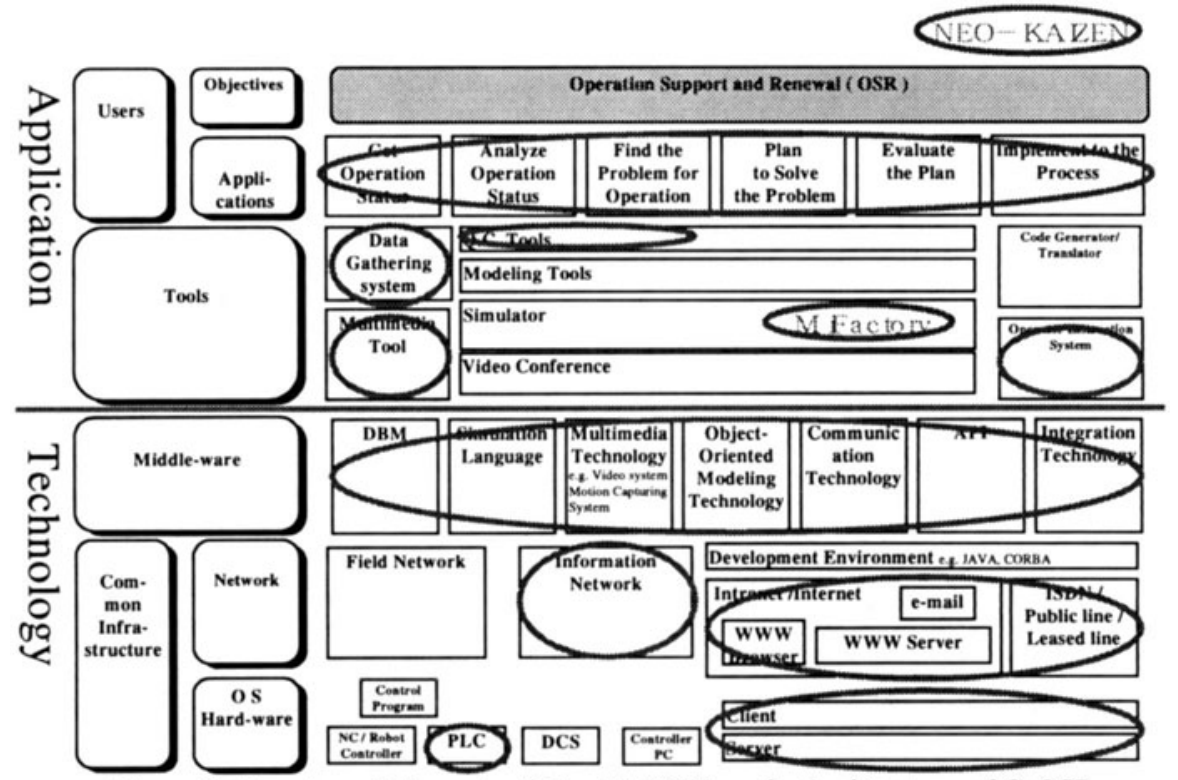

Figure 2 Mapping of elements of Neo-KAIZEN on the Architecture of G-OSR

Primarily, the G-OSR architecture is a matrix, within which groups of tools are classified as 'Technology' consisting of Middle-ware and Common Infrastructure on the vertical axis and 'Application' consisting of Users and Tools on the horizontal axis. On the horizontal axis, for instance, groups are classified into applications equivalent to each phase of the spiral approach of Neo-KAIZEN. Figure 2 shows the mapping of the groups of tools developed in Neo-KAIZEN systems to the G-OSR architecture. Common use of the 
information infrastructure and middle-ware enables to design and build the KAIZEN systems easily in the global environment. Low cost and rapid design of the KAIZEN systems can be realized by the G-OSR architecture.

\section{EXPERIMENTAL DEMONSTRATION SYSTEMS}

The Neo-KAIZEN spiral approach model was applied to the three experimental demonstrations of (1) operation improvement of an electronics product assembly, (2) quality improvement on an automated assembly and (3) improvement on a continuous process plant.

\subsection{Operation improvement}

The proposed Neo-KAIZEN system has been evaluated by the data from an U-shape assembly line in OMRON Corp. The assembly line of electronic equipment is shown in Figure 3. Over two hundred kinds of products are produced by the groups of two or three workers in the line. Their operations change dynamically depending on the model type or the batch size to be assembled. Usually the change for operation balancing is required. The system monitors the operation status, gathers the status data and stores the data to the database as time synchronized data [6]. Then, an improvement item is quickly proposed by the system.

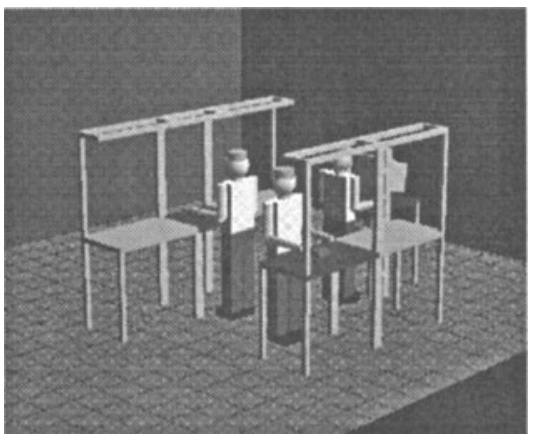

Virtual simulation model

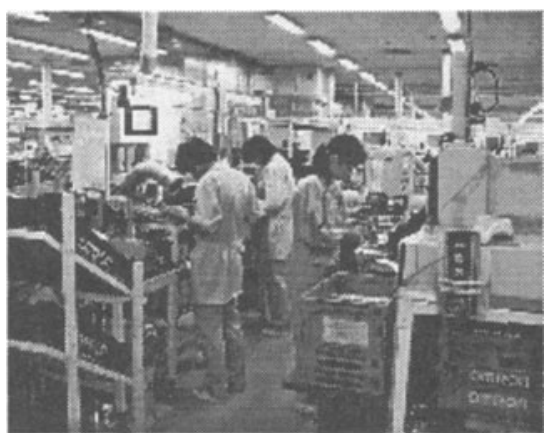

Real Shop floor

Figure $3 \quad U$-shape assembly line

In this system, POP (Point of production) system and video camera monitor the workers for getting status. The system stored the logging data in the Web-based Synchronous Data Logger. The logged data is analysed by QCAS (a QC assistant tool). Then, a small group of engineers and workers make a solution for improving their operations. The solution can be evaluated by MiFactory (a simulation tool) before implementing to the real shop floor. After the evaluation, the Operator Instruction System informs the 
operation manual to workers using multi-media. We could get the successful result from this application.

An improvement scenario paying attention to worker's performance is described as a demonstration system according to the following steps.

(1) Record video images of the process if deviation from the pitch time exceeds $30 \%$.

(2) Analyse causes of referring to the image.

(3) Enter data obtained from the analysis into the simulator model and make simulation to see if a relationship with worker's skill exists.

(4) Through simulation find out such work combinations and number of workers that promise higher work efficiency.

(5) Modify the work procedure.

(6) The result: As to the work that is analysed, time including preparatory time is reduced. After the KAIZEN activity is performed, the system performance is improved.

\subsection{Quality improvement}

The second application is improvement of yield of PCB (Printed Circuit Board) assembly operations in an automated assembly line.

(i) Target

In the inspection process of the PCB assembly line, an automatic machine solders parts accurately at a specified position, and the inspection equipment checks the soldering work.

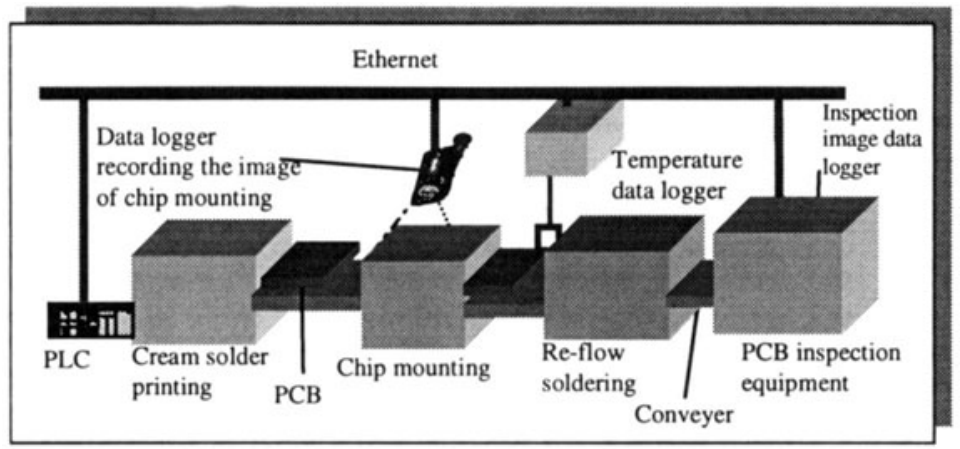

Figure 4 Configuration of the information system of $P C B$ assembly

(ii) Tasks for improvement

The goals are to reduce the number of defective soldering when the line is in operation and to increase the assemblies yield.

(iii) Required information for improvement

- Visually check the soldered appearance. 
- Check the shape, the height and the quantity of solder.

- Deviation in the quantity of solder can be determined from checks of the soldered area.

- Low Solder temperature.

- Defect of timing of chip mounting and wrong parts.

(iv) Improvement steps

An improvement demonstration scenario is described according to the following steps.

(1) Collection of primary information mainly obtained as data from the inspection equipment ascertains the real cause. (2) To establish the cause, collection of detailed information is realized using a data logger. When the inspection result is NG, solder temperature, video image of chip mounting and surface image of solder are recorded. (Collection of secondary information)

(3) Soldering process specialists are employed for the analysis of causal factors and solve the problems easily using the information from the automated assembly line.

(4) The result: The information gathering and editing time is reduced to half by the support system.

\subsection{Demonstration in Continuous Process Plant}

A Neo-KAIZEN system was implemented to a continuous process plant for a demonstration.

(i) Objectives

A chemical plant located in Asia was used as the target, and a demonstration was carried out with the following points as objectives.

- To support for site operation troubles from the design side.

- To support checking, inspection, and maintenance of equipment.

- Actual plant operation data to be analysed, and the data used in the next design such as process improvement or equipment specification review.

- To support for management works such as production management or equipment management.

(ii) Configuration

To accomplish above objectives (i), the system was reviewed using the configuration shown in Figure 5. The study concentrated on (a) data collection, (b) data transfer, and (c) some data analysis and evaluation.

(iii) The result

Experiment with the demonstration system configuration was applied at an existing overseas plant. It was confirmed that the collection and transfer of the data through the Internet could be carried out automatically. The system has now entered the stage of operational test. 


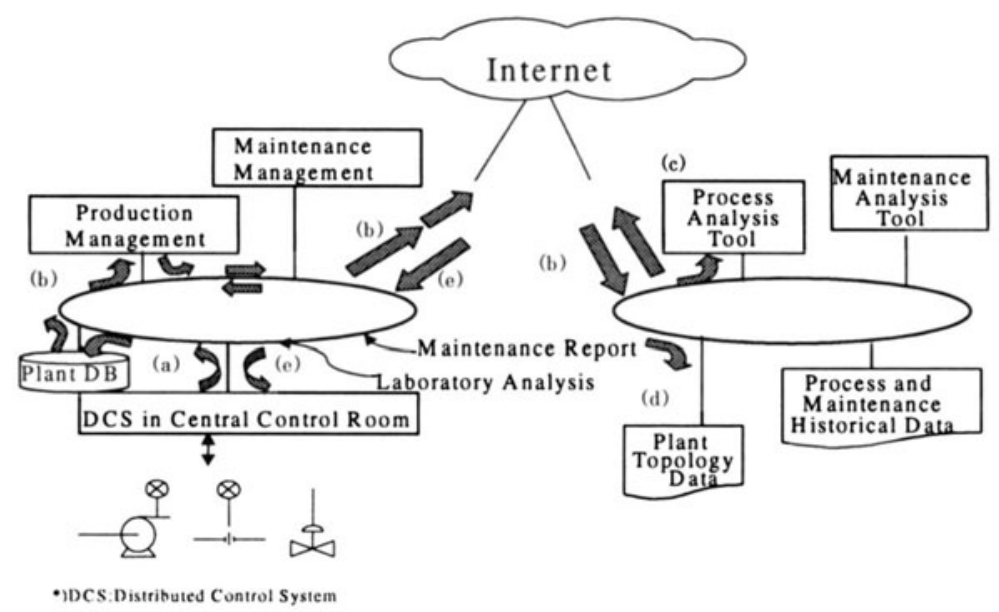

Figure 5 System Configuration of a Continuous Process Plant for Demonstration

\section{CONCLUSIONS}

The Neo-KAIZEN systems based on the spiral approach model were proposed. Three applications of demonstration systems using the data from some plants were designed. It has been verified that the Neo-KAIZEN systems on the G-OSR information architecture are feasible and can efficiently perform the KAIZEN activity on operating electronics assembly lines and a chemical plant. These systems will be easily designed on the GOSR information architecture in the future. We will continue to study the methodology of design for the G-OSR systems.

\section{REFERENCES}

[1] Goto, H., et.al.,(1997). "Remote Renewal By Aggregating Real and Virtual Models In Manufacturing Systems”, Proceedings of IMS '97, pp.77-81, IFAC.

[2] Mitsuishi, M., et.al.,(1995). Proceedings of IROS'95, pp.13-20, IEEE.

[3] Iwata, K., et.al.,(1995). Proceedings of EI'95, pp.154-167.

[4] Hosotani, K.,(1992). The QC Problem Solving Approach - Solving Workplace Problems the Japanese Way, ISBN4-906224-91-1 C0034.

[5] Ollus,M.,et.al.(1998). "Systems for Support of Process Operations", Proceedings of IiM98.

[6] Mori, K.,(1998). "Computer Aided Continuous Improvement (KAIZEN) System on Operating the Manufacturing System”, Proceedings of PTK98, pp.375-380, IPK. 